\title{
Design of Hartmann Null Screens for Testing Plane-convex Aspheric Lens
}

\author{
Gabriel Castillo-Santiago, Diana Castán-Ricaño, Maximino Avendaño-Alejo \\ Universidad Nacional Autónoma de México, \\ Centro de Ciencias Aplicadas y Desarrollo Tecnológico, \\ México
}

\{Gabriel Castillo-Santiago, Diana Castán-Ricaño, maximino.avendano\}@ccadet.unam.mx

\begin{abstract}
A new method to design Hartmann null screens for testing aspheric lenses is presented. It is based on the exact ray tracing equation, consider a plane wavefront impinging on the lens.
\end{abstract}

Keywords: Hartmann null screens, aspheric lens, testing.

\section{Introduction}

A spot diagram are points representing the intersections of rays from a point object given within a focal plane of a lens, on the other hand an experimental procedure similar to spot diagram is the Hartmann test in order to evaluate lenses.

For us, the null test involve simplicity, because it is straightforward to recognize a predefined pattern of spots. Some examples for Null test were described by reflection in [1] or refraction [2], but in the case of lenses with arbitrary aspheric terms as far as we know, there is no analysis by using null tests by refraction in both plane-convex and convex-plane configuration.

\section{Designing Hartmann Null Screen}

We define that the $\mathbf{Z}$ axis is parallel to the optical axis and we assume that the $\mathbf{Y}-\mathbf{Z}$ plane is the plane of incidence, there is rotational symmetry about the $\mathbf{Z}$ axis. We consider ideally a bundle of rays propagating parallel to the optical axis, crossing the aspheric surface of the lens having two refractions, the first is represented by $(\mathrm{PaPi})$, and the second refraction it is produced at the plane surface, it is represented by (PiPO) as is shown in Fig. 1(a).

Let $\mathrm{h}$ be the height of every incident ray, $\mathrm{t}$ is the thickness of the lens, ni the index of refraction of the lens for a predefined wavelength which is immersed in a medium with index of refraction $n a,(n i>n a)$, and where we have assumed that $S_{h_{N}}$ represents the aspheric equation in a meridional plane which is given by 


$$
S_{h_{N}}=\frac{c h^{2}}{1+\sqrt{1-(k+1) c^{2} h^{2}}}+\sum_{i=1}^{N} A_{2 i} h^{2 i},
$$

where $\mathrm{c}=1 / \mathrm{R}$ is the paraxial curvature, $k$ is the conic constant, $\mathrm{A}_{2 \mathrm{i}}$, are the aspheric order terms with $N$ the number of aspheric terms included in the polynomial. According to reference [3] the equation for $\left(P_{i} P_{0}\right)$ refracted outside of the aspheric lens be expressed by

$$
y=h-\frac{\left(n_{i}^{2}-n_{a}^{2}\right)\left(t-S_{h_{N}}\right) S_{h_{N}}^{\prime}}{n_{i}^{2}+n_{a} \Lambda}-\frac{\left(n_{i}^{2}-n_{a}^{2}\right)(z-t) S_{h_{N}}^{\prime}}{\sqrt{n_{a}^{2}\left(n_{a}+\Lambda\right)^{2}-\left(n_{i}^{2}-n_{a}^{2}\right)^{2} S_{h_{N}}^{2}}},
$$

where:

$S_{h_{N}}$ is the first derivate of Eq. (1) with respect to $h$, and we have redefined $\Lambda=$ $\left[n_{i}^{2}+\left(n_{i}^{2}-n_{a}^{2}\right) S_{h_{N}}^{2}\right]^{1 / 2}$

To calculate the positions of the points on the Hartmann null screens, we follow all the steps well explained in [1], starting at the detection plane which has a predefined size of a CCD sensor, then the coordinates of the point pi in the aspheric surface are not selected a priori but must be calculated giving an arbitrary point $p_{0}=\left(z_{0}, y_{0}\right)$ at the detection plane, and using the exact ray tracing equation as is shown in Fig.1(b).

In this work we design Null screens for both, plane-convex and convex-plane configurations, the complete equations are well explained in reference [3], the detection plane has been located in the paraxial plane in order to evaluate the spherical aberration in aspheric lenses which theory is described in [4]. In others words, by substituting $\mathrm{y} \rightarrow$ $\mathrm{y} 0$ and $\mathrm{z} \rightarrow \mathrm{z} 0$ in to Eq. (2) and solving for $h$ we obtain the values for $h_{i}$ which will form the null screens to test the lens

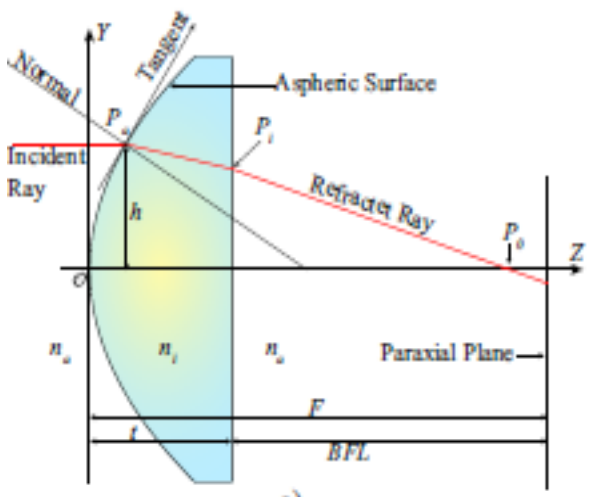

a)

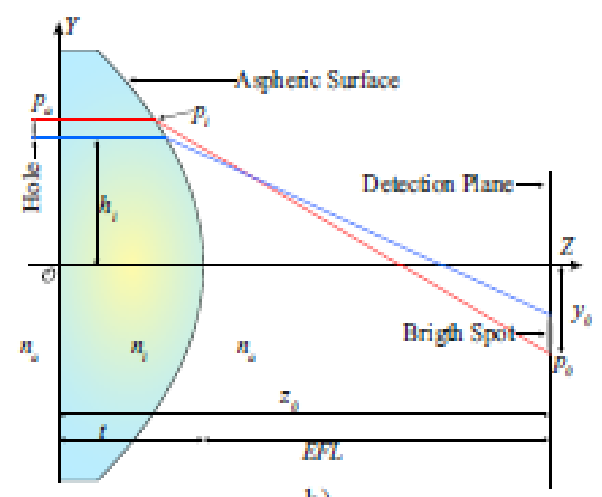

b)

Fig. 1. a) Process of refraction produced by an aspheric lens, and their associated parameters in the convex-plane configuration. b) Process to design null screens in a plane-convex configuration aspheric lens. 


\section{Qualitative Evaluation}

In this work we have evaluated qualitatively a lens with $\mathrm{F} / \#=0.86$, by using the following parameters: $n_{a}=1, n_{i}=1.523$ for $\lambda=780 \mathrm{~nm}, R=13.8595 \mathrm{~mm}, k=-1, t=$ $11.04 \mathrm{~mm}$, diameter $D=30 \mathrm{~mm}$, and entrance aperture $H= \pm D / 2$, these values correspond to item ACL3026 from Thorlabs. The diagram of the experimental setup is show in Fig. 2, where two polarizers can be seen, which have been used to reduce the intensity of the laser beam on the CCD sensor, the screens have been printed on acetate foils using a photographic printer specified at 150 lines. The CCD sensor has an active area of $\mathrm{A}=6.6 \mathrm{~mm} \times 8.8 \mathrm{~mm}$ and $480 \times 640$ pixels respectively. The light source is a He$\mathrm{Ne}$ laser with spatial filtering and a collimated beam, where a collimator lens with F/\# $=6$ has been implemented to reduce the central hot spot by increasing the distance between the collimator lens and the pinhole.

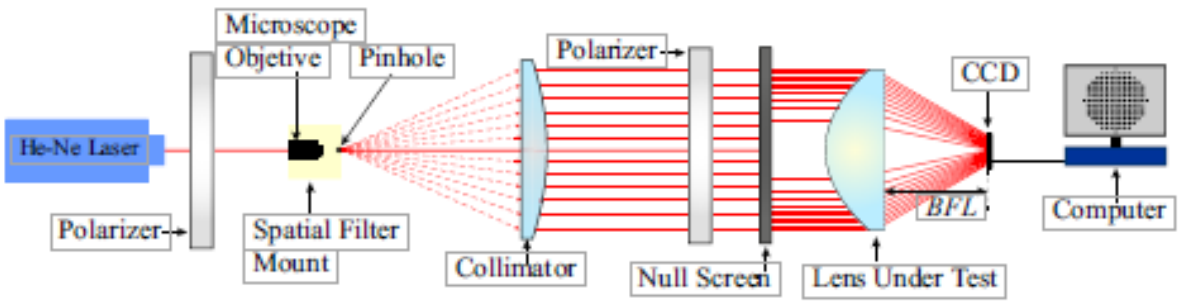

Fig. 2. Diagram of the experimental setup to test a plane-convex aspherical lens using null screens.

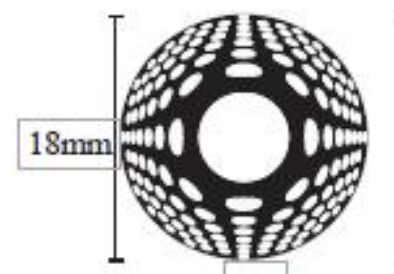

1a)

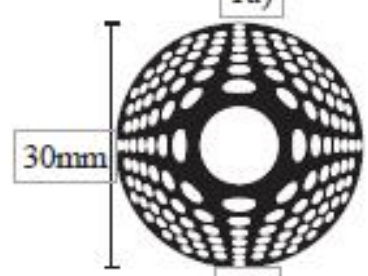

1b)

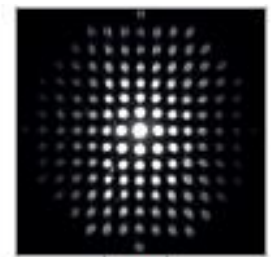

2a)

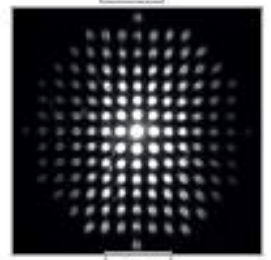

2b)

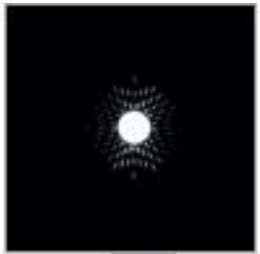

3a)

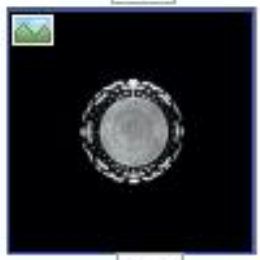

3b)

Fig. 3. 1a) Null screen with 15 drops in the central line for plane-convex configuration, 2a) Image recorded on the CCD at the focal length, 3a) Image recorded out of the plane of design. 1b) Null screen for convex-plane configuration $2 \mathrm{~b}$ ) Uniform array recorded at the CCD in the back focal length, 3. Image recorded inside the caustic region.

It is important to state that if visual observations are made in real time, the computed spots used as null screen can be calculated to produce an observable pattern of adequate 
size for the lens under test at a predefined detection plane as are show in Fig. 3, the non-uniformity of intensity recorded on the CCD sensor such as are shown in Fig. 3 (2) could be produced by several factors, but a graduated filter at the collimator, or even as part of the null screen, can be used as a possible solution to obtain a uniform intensity distribution of the incident beam on the CCD sensor.

In plane-convex configuration we can observe that the null screen has a smaller coverage in the entrance aperture than convex-plane configuration, it is due to that the marginal rays undergo total internal reflection as is show in Fig. 3 (1a and 1b), diffraction effects can be seen in some drops due to small size of the holes of the null screens. In Fig. 3 (2b) the images for convex-plane configuration show less diffraction effects than plane-convex configuration, but a high mechanical accuracy is required.

\section{Conclusions}

A simple method to design null screens in order to test by refraction either plane-convex or convex-plane configuration aspheric lenses has been presented by using drops arrays which were printed on acetate foil by using a photographic printed specified at 150 lines. The null screens in convex-plane configuration coverage more area than planeconvex configuration. This method can be used to evaluate quantitatively the spherical aberration, furthermore to reconstruction of the shape of the surface.

Acknowledgments. This work has been partially supported by CONACYT (\#168570) and PAPIIT-UNAM (\#IN114414).

\section{References}

1. Rufino Díaz-Uribe and Manuel Campos-García, "Null-screen testing of a fast convex aspheric surfaces," Appl. Opt., 39, 2670-2677 (2000).

2. M. Avendaño-Alejo, Dulce González-Utrera, Naser Qureshi, Luis Castañeda, "Null RonchiHartmann test for a lens," Opt. Exp., 18, 21131-21137 (2010).

3. G. Castillo-Santiago, "Design and evaluation of physical properties for fast plane-convex aspheric lenses," M. Eng. dissertation in process (UNAM, 2014).

4. Castillo-Santiago G., Avendaño-Alejo M., Díaz-Uribe R., Castañeda L."Analytic aspheric coefficients to reduce the spherical aberration of lens elements used in collimated light," Appl. Opt., 53, pp.4939-4946 (2014). 